\title{
On the Use of Computable Features for Film Classification
}

\author{
Zeeshan Rasheed, Yaser Sheikh, Student Member, IEEE, and Mubarak Shah, Fellow, IEEE
}

\begin{abstract}
This paper presents a framework for the classification of feature films into genres, based only on computable visual cues. We view the work as a step toward high-level semantic film interpretation, currently using low-level video features and knowledge of ubiquitous cinematic practices. Our current domain of study is the movie preview, commercial advertisements primarily created to attract audiences. A preview often emphasizes the theme of a film and hence provides suitable information for classification. In our approach, we classify movies into four broad categories: Comedies, Action, Dramas, or Horror films. Inspired by cinematic principles, four computable video features (average shot length, color variance, motion content and lighting key) are combined in a framework to provide a mapping to these four highlevel semantic classes. Mean shift classification is used to discover the structure between the computed features and each film genre. We have conducted extensive experiments on over a hundred film previews and notably demonstrate that low-level visual features (without the use of audio or text cues) may be utilized for movie classification. Our approach can also be broadened for many potential applications including scene understanding, the building and updating of video databases with minimal human intervention, browsing, and retrieval of videos on the Internet (video-on-demand) and video libraries.
\end{abstract}

Index Terms-High-key, low-key, movie genres, previews, shot length, video-on-demand.

\section{INTRODUCTION}

$\mathbf{F}$ ILMS are a means of expression. Directors, actors, and cinematographers use this medium as a means to communicate a precisely crafted storyline. This communication operates at several levels; explicitly, with the delivery of lines by the actors, and implicitly, with the background music, lighting, camera movements and so on. Directors often follow well-established rules, commonly called "film grammar" in literature, to communicate these concepts. Like any natural language, this grammar has several dialects, but is more or less universal. This fact in filmmaking (as compared to arbitrary video data) suggests that knowledge of cinematic principles can be exploited effectively for the understanding of films. To interpret an idea using the grammar, we need to first understand the symbols, as in natural languages, and second, understand the rules of combination of these symbols to represent concepts. D. Arijon, a famous name in film literature, writes, "All the rules of film grammar have been on the screen for a long time. They are used by filmmakers as far apart geographically and in style as Kurosawa in Japan, Bergman in Sweden, Fellini in Italy, and Ray in

Manuscript received January 20, 2002; revised November 18, 2002. This paper was recommended by Associate Editor F. Pereira.

The authors are with the Department of Computer Science, University of Central Florida, Orlando, FL 38216-2362 USA (e-mail: zrasheed@ cs.ucf.edu).

Digital Object Identifier 10.1109/TCSVT.2004.839993
India. For them, and countless others this common set of rules is used to solve specific problems presented by the visual narration of a story" [3, p. 4].

In order to exploit knowledge of these ubiquitous techniques, it is necessary to be able to relate the symbols of film grammar to computable video features. Computable video features, as the name suggests, are defined as any statistic of the available video data. Since the relationship between film grammar symbols and high-level film semantics is known, if we are able to find computable representations of these symbols, the problem of film classification can be favorably posed. Unfortunately, not all the symbols of film grammar can be well-represented in terms of a statistic. For instance, how does one compute the irony in a scene? It is immediately evident that high-level symbols like emotion, irony, or gestures are difficult to represent as statistics. On the other hand, low-level symbols like lighting, shot length and background music are far easier to represent. It should also be noted that low-level symbols correspond to the implicit communication that the director uses and, incidently, are also the type of symbols that have the most established techniques. Audiences too become "trained" to interpret low-level symbols in a certain way, as is evidenced by feelings of expectation associated with silence, or feelings of fear associated with dim lighting. These ideas are investigated in depth in [24], [3].

Films constitute a large portion of the entertainment industry. Every year about 4500 films are released around the world, which correspond to approximately 9000 hours of video [28]. While it is feasible to classify films at the time of production, classification at finer levels, for instance classification of individual scenes, would be a tedious and substantial task. Currently, there is a need for systems to extract the "genre" of scenes in films. Application of such scene-level classification would allow departure from the prevalent system of movie ratings to a more flexible system of scene ratings. For instance, a child would be able to watch movies containing a few scenes with excessive violence, if a prefiltering system can prune out scenes that have been rated as violent. Such semantic labeling of scenes would also allow far more flexibility while searching movie databases. For example, automatic recommendation of movies based on personal preferences could help a person choose a movie, by executing a scene level analysis of previously viewed movies. While the proposed method does not actually achieve scene classification, it provides a suitable framework for such work.

Some justification must be given for the use of previews for the classification of movies. Since movie previews are primarily commercial advertisements, they tend to emphasize the theme of the movie, making them particularly suited for the task of 


\section{Categorization by Human Observers}

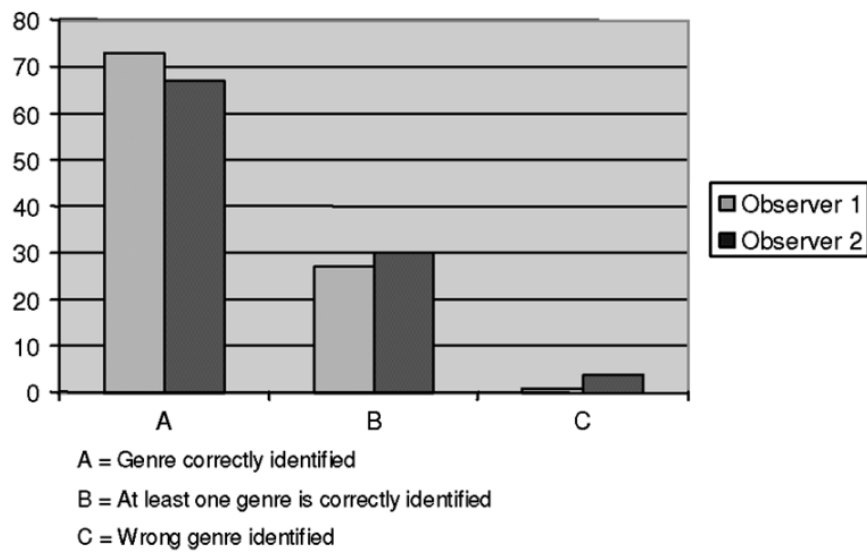

Fig. 1. Genre classification by human observers with respect to the ground truth obtained from the IMDB and Apple website. Observers were asked to categorize films into four genres based on their previews.

genre classification. For example, previews of action movies inevitably contain shots of fights, chases, and sometimes crashes, explosions and gunfire. Exceptions exist, of course, and in order to strengthen the claim that high-level semantic classification based on previews is possible, we conducted human evaluations of our data set consisting of over a hundred film previews and compared the results with the ground truth obtained from the Internet Movie Database (IMDB) [4] and the Apple website, [2]. Two observers were asked to watch the previews and classify each movie into the four genres. Both observers managed to identify at least one of the genres in the ground truth, for practically all the movies. Ignoring bias of prior knowledge, what the experiment suggested was that classification based on movie previews is, at the very least, possible. The results of the evaluation are displayed in Fig. 1. In conclusion, we present a framework for genre classification based on four computed features, average shot length, color variance, motion content and lighting key, from film previews. It is noteworthy that in this work inferences are made using visual features only, and no audio or textual information is used. Furthermore, since both previews and scenes are composed of several shots, this framework can be suitably extended for applications of scene classification.

The rest of the paper is organized as follows. Related work is discussed in Section II. In Section III, we present the computable video features that are used for classification in our work. Section IV details the use of mean shift classification as a clustering approach in our application. A discussion of the results is presented in Section V, followed by conclusions in Section VI.

\section{RELATED WORK}

One of the earliest research efforts in the area of video categorization and indexing was the Informedia Project [13] at Carnegie Mellon University. It spearheaded the effort to segment and automatically generate a database of news broadcasts every night. The overall system relied on multiple low-level cues, like video, speech, close-captioned text, and other cues. However, there are a few approaches which deal with higher level semantics, instead of using low-level feature matching as the primary indexing criteria. Work by Fischer et al. in [11] and a similar approach by Truong et al. in [26], distinguished between newscasts, commercials, sports, music videos, and cartoons. The feature set consisted of scene length, camera motion, object motion and illumination. These approaches were based on training the system using examples and then employing a decision tree to identify the genre of the video.

Content based video indexing also constitutes a significant portion of the work in this area. Chang et al. [7] developed an interactive system for video retrieval. Several attributes of video such as color, texture, shape, and motion were computed for each video in the database. The user was required to provide a set of parameters for attributes of the video that was being searched for. These parameters were compared with those in the database using a weighted distance formula for the retrieval. A similar approach has also been reported by Deng et al. [9].

The use of hidden Markov models (HMMs) has been very popular in the research community for video categorization and retrieval. Naphade et al. [19] proposed a probabilistic framework for video indexing and retrieval. Low-level features were mapped to high-level semantics as probabilistic multimedia objects called multijects. A Bayesian belief network, called a multinet, was developed to perform semantic indexing using HMMs. Some other examples that make use of probabilistic approaches are [6], [10], and [29]. Qian et al. also suggested a semantic framework for video indexing and detection of events. They presented an example of hunt detection in videos, [23].

A large amount of research work on video categorization has also been done in the compressed-domain using MPEG-1 and MPEG-2. The work in this area utilizes the extractable features from compressed video and audio. Although the compressed information may not be very precise, it avoids the overhead of computing features in the pixel domain. Kobla et al. [16] used discrete cosine transform (DCT) coefficients, macroblock, and motion vector information of MPEG videos for indexing and retrieval. Their proposed method was based on Query-by-Example and found the spatial (DCT coefficients) and temporal (motion) similarities among the videos using FastMap. The methods proposed in [20] and [30] are a few more examples which also work on compressed video data. Lu et al. [17] applied an HMM based approach in the compressed domain and promising results were presented. Recently, the MPEG-7 community has focused on video indexing by using embedded semantic descriptors, [5]. However, the standardization of MPEG-7 is currently under development and the content-to-semantic interpretation for retrieval of videos is still an open question for the research community.

Specific to film classification, Vasconcelos et al. proposed a feature-space based approach in [27]. In this work, two features of the previews, average shot length and shot activity, were used. In order to categorize movies they used a linear classifier in the two-dimensional (2-D) feature space. An extension of their approach was presented in Nam et al.[18], which identified violence in previews. They attempted to detect violence using audio and color matching criteria. One problem with these existing approaches in film classification is the crude structure that is imposed while classifying data (in the form of the linear classifier). In our work, we adopt a nonparametric approach, using 
mean shift clustering. Mean shift clustering has been shown to have excellent properties for clustering real data. Furthermore, we exploit knowledge of cinematic principles, presenting four computable features for the purposes of classification. We believe that the extendibility of the proposed framework to include new, possibly higher level features is an important aspect of the work. Since the approach discovers the structure of the mapping between features and classes autonomously, the need to handcraft rules of classification is no longer required.

\section{COMPUtABle VideO FEATURES}

In this paper, we present the problem of semantic classification of films within the feature-space paradigm. In this paradigm, the input is described through a set of features that are likely to minimize variance of points within a class and maximize variance of points across different classes. A parametric representation of each feature is computed and is mapped to a point in the multidimensional space of the features. Of course, the performance depends heavily on the selection of appropriate features. In this section, we present four computable features that provide good discrimination between genres. An (arguably) comprehensive list of genres can be found at http://us.imdb.com/Sections/Genres/, which enumerates them as Action, Adventure, Animation, Comedy, Crime, Documentary, Drama, Family, Fantasy, Film Noir, Horror, Musical, Mystery, Romance, Science Fiction, Short, Thriller, War, and Western. From this list, we identified four major genres: Action, Comedy, Horror, and Drama. There are two reasons for this choice. First, these genres represent the majority of movies currently produced, and most movies can be classified, albeit loosely, into at least one of these major genres. Second, we have selected these four genres since it is between these genres that low-level discriminant analysis is most likely to succeed. In other words, it is in these genres that we propose correlation exists between computable video features and their respective genres. However, the data set itself was not prescreened to fit specifically into one of these genres, as the subsequent results will show, many movies fit more than one of the categories. Rather than espouse individual genre classification, we acknowledge the fact that a film may correctly be classified into multiple genres. For instance, many Hollywood action films produced these days have a strong element of comedy as well. In the remainder of this section, we discuss the four features that are employed for classification, namely average shot length, shot motion content, lighting key, and color variance.

\section{A. Shot Detection and Average Shot Length}

The first feature we employ is the average shot length. This feature was first proposed by Vasconcelos in [27]. The average shot length as a feature represents the tempo of a scene. The director can control the speed at which the audience's attention is directed by varying the tempo of the scene, [1]. The average shot length provides an effective measure of the tempo of a scene, and the first step in its computation is the detection of shot boundaries. A shot is defined as a sequence of frames taken by a single camera without any major change in the color content of consecutive images. Techniques based on color histogram comparison have been found to be robust and are used by several researchers for this purpose. In our approach, we extend the algorithm reported in [12] for the detection of shot boundaries using color histogram intersection in the HSV space, where $\mathrm{H}$ stands for hue or color, $\mathrm{S}$ stands for saturation or precentage of white, and $\mathrm{V}$ stands for value or brightness.

Each histogram consists of 16 bins-eight for the hue, four for the saturation, and four for the value components of the HSV color space. Let $S(i)$ represent the intersection of histograms $H_{i}$ and $H_{i-1}$ of frames $i$ and $i-1$, respectively. That is

$$
S(i)=\sum_{j \in \text { all bins }} \min \left(H_{i}(j), H_{i-1}(j)\right) .
$$

The magnitude $S(i)$ is often used as a measure of shot boundary in related works. The values of $i$ where $S(i)$ is less than a fixed threshold are assumed to be the shot boundaries. This approach works quite well (see [12]) if the shot change is abrupt and there are no shot transition effects (wipes, dissolves etc). Previews are generally made with a variety of shot transition effects. We have observed that the most commonly used transition effect in previews is a dissolve in which several frames of consecutive shots overlap. Applying a fixed threshold to $S(i)$ when the shot transition occurs with a dissolve generates several outliers because consecutive frames differ from each other until the shot transition is completed.

To improve the accuracy, an iterative smoothing of the one dimensional function $S$ is performed first. We have adapted the algorithm proposed by Perona et al. [22] based on anisotropic diffusion. This is done in the context of scale space. $S$ is smoothed iteratively using a Gaussian kernel such that the variance of the Gaussian function varies with the signal gradient. Formally

$$
S^{t+1}(i)=S^{t}(i)+\lambda\left[c_{E} \cdot \nabla_{E} S^{t}(i)+c_{W} \cdot \nabla_{W} S^{t}(i)\right]
$$

where $t$ is the iteration number and $0<\lambda<1 / 4$ with

$$
\begin{gathered}
\nabla_{E} S(i) \equiv S(i+1)-S(i) \\
\nabla_{W} S(i) \equiv S(i-1)-S(i) .
\end{gathered}
$$

The condition coefficients are a function of the gradients and are updated for every iteration

$$
\begin{gathered}
c_{E}^{t}=g\left(\left|\nabla_{E} S^{t}(i)\right|\right) \\
c_{W}^{t}=g\left(\left|\nabla_{W} S^{t}(i)\right|\right)
\end{gathered}
$$

where $g\left(\nabla_{E} S\right)=e^{-\left(\frac{\left|\nabla_{E}\right|}{k}\right)^{2}}$ and $g\left(\nabla_{W} S\right)=e^{-\left(\frac{\left|\nabla_{W}\right|}{k}\right)^{2}}$. In our experiments, the constants were set to $\lambda=0.1$ and $k=0.1$. Finally, the shot boundaries are detected by finding the local minima in the smoothed similarity function $S$. Thus, a shot boundary will be detected where two consecutive frames will have minimum color similarity. This approach reduces the false alarms produced by the fixed threshold method.

Fig. 2 presents a comparison between the two methods: Fig. 2(a) using a fixed threshold method and (b) using the proposed method. The similarity function $S$ is plotted against the frame numbers. Only the first 400 frames are shown for convenient visualization. There are several outliers in Fig. 2(a) because gradually changing visual contents from frame to 


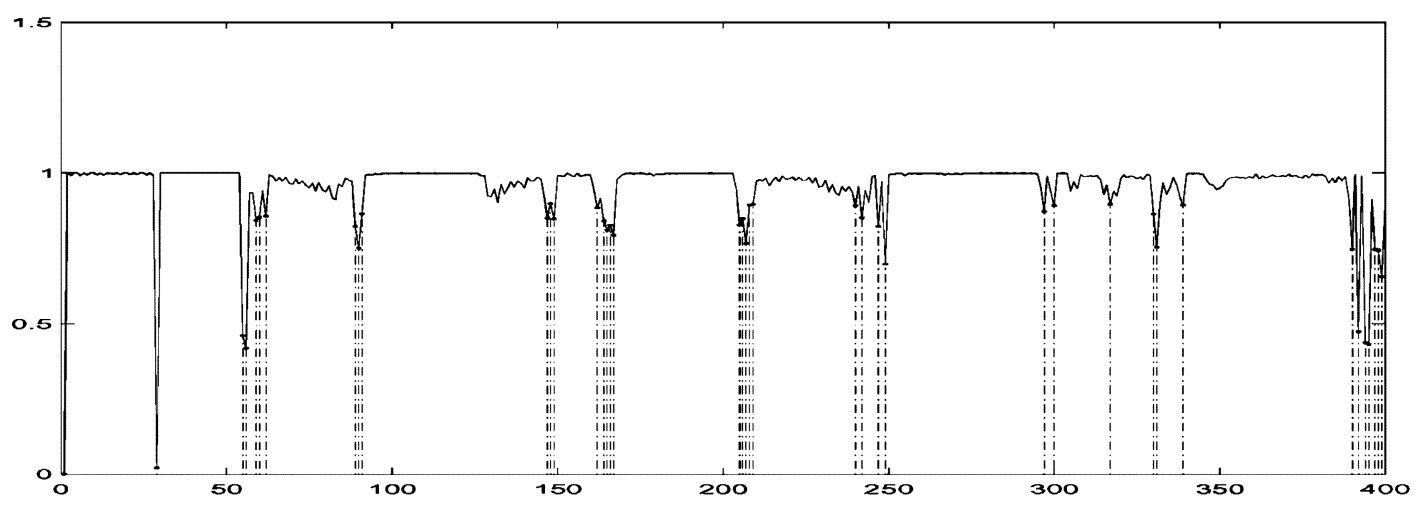

(a)

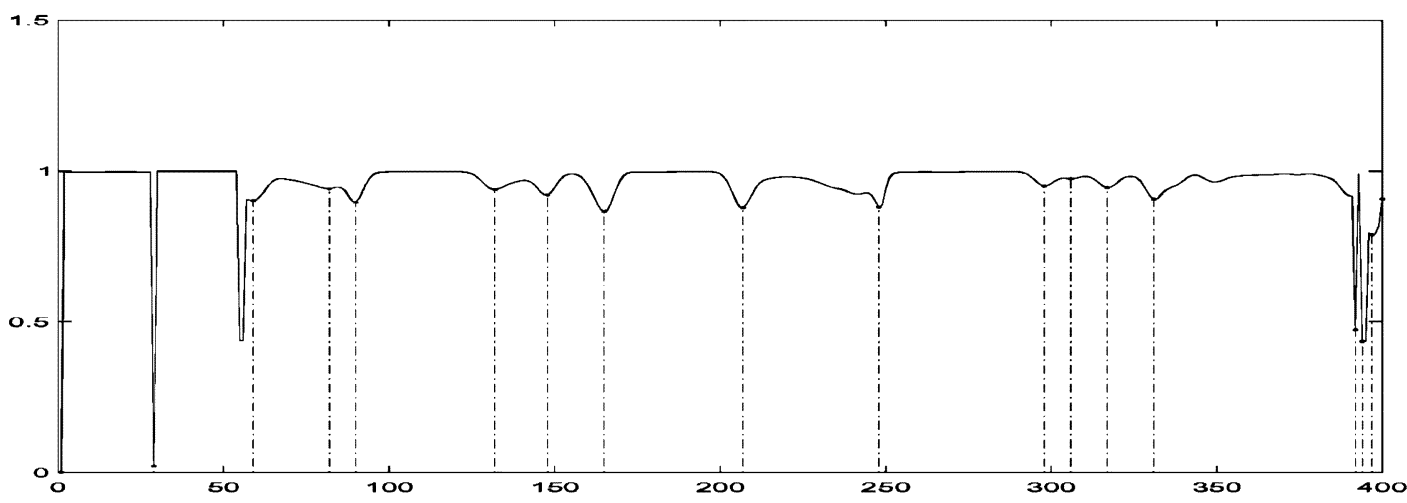

(b)

Fig. 2. (a) Shot detection using fixed threshold method for a segment of the movie trailer of Red Dragon. Only first 400 frames are shown for convenient visualization. There are 17 shots identified by a human observer. (a) Fixed threshold method. Vertical lines indicate the detection of shots. Number of shots detected: 40; Correct: 15; False positive: 25; False negative: 2 (b) Shots detected by proposed method. Number of shots detected: 18; Correct: 16; False positive: 2; False negative: 1.

frame (the dissolve effect) are detected as a shot change. For instance, there are multiple shots detected around frame numbers 50, 150, and 200. However, in Fig. 2(b), a shot is detected when the similarity between consecutive frames is minimum. Compare the detection of shots with Fig. 2(a). Fig. 3 shows improved shot detection for the preview of Road Trip. See Table I that lists precision and recall of shot detection for some of the trailers in the data set.

The average shot length is then computed for each preview. This feature is directly computed by dividing the total number of frames by the total number of shots in the preview (the statistical mean). Our experiments show that slower paced films such as dramas have larger average length as they have many dialogue shots, whereas action movies appear to have shorter shot lengths because of rapidly changing shots. Each detected shot is represented by a key frame to analyze the shot's color attributes. We use the middle frame of each shot as the key frame.

\section{B. Color Variance}

Zettl observes in, [31], "The expressive quality of color is, like music, an excellent vehicle for establishing or intensifying the mood of an event." In this work, we are interested in exploiting the variance of color in a clip as a whole to discriminate between genres of a film. Intuitively, the variance of color has a strong correlational structure with respect to genres, as it can be seen, for instance, that comedies tend to have a large variety of bright colors, whereas horror films often adopt only darker hues. Thus, in order to define a computable feature two requirements have to be met. First, a feature has to be defined that is global in nature, and second, distances in the color space employed should be perceptually uniform. We employ the CIE Luv space, which was designed to approach a perceptually uniform color space. To represent the variety of color used in the video we employ the generalized variance of the Luv color space of each preview as a whole. The covariance matrix of the multivariate vector (three-dimensional in our case) is defined as

$$
\rho=\left[\begin{array}{ccc}
\sigma_{L}^{2} & \sigma_{L u}^{2} & \sigma_{L v}^{2} \\
\sigma_{L u}^{2} & \sigma_{u}^{2} & \sigma_{u v}^{2} \\
\sigma_{L v}^{2} & \sigma_{u v}^{2} & \sigma_{v}^{2}
\end{array}\right] .
$$

The generalized variance is obtained by finding the determinant of (5)

$$
\sum=\operatorname{det}(\rho)
$$

This feature is used as a representation of the color variance. All key frames present in a preview are used to find this feature.

\section{Motion Content}

The visual disturbance of a scene can be represented as the motion content present in it. The motion content represents the amount of activity in a film. Obviously, action films would have 


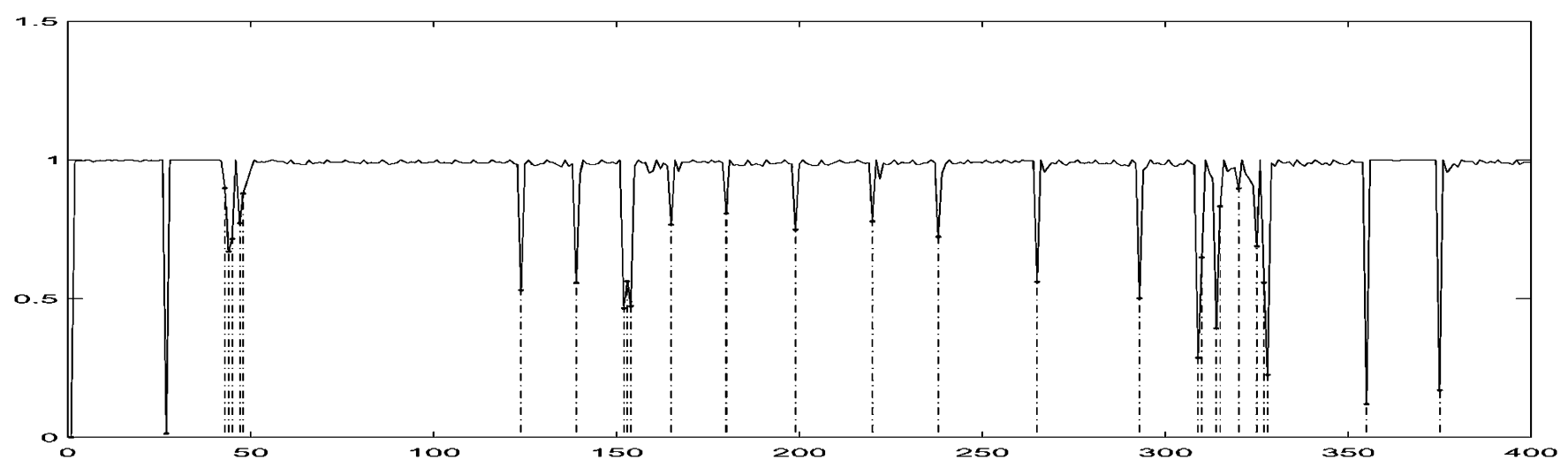

(a)

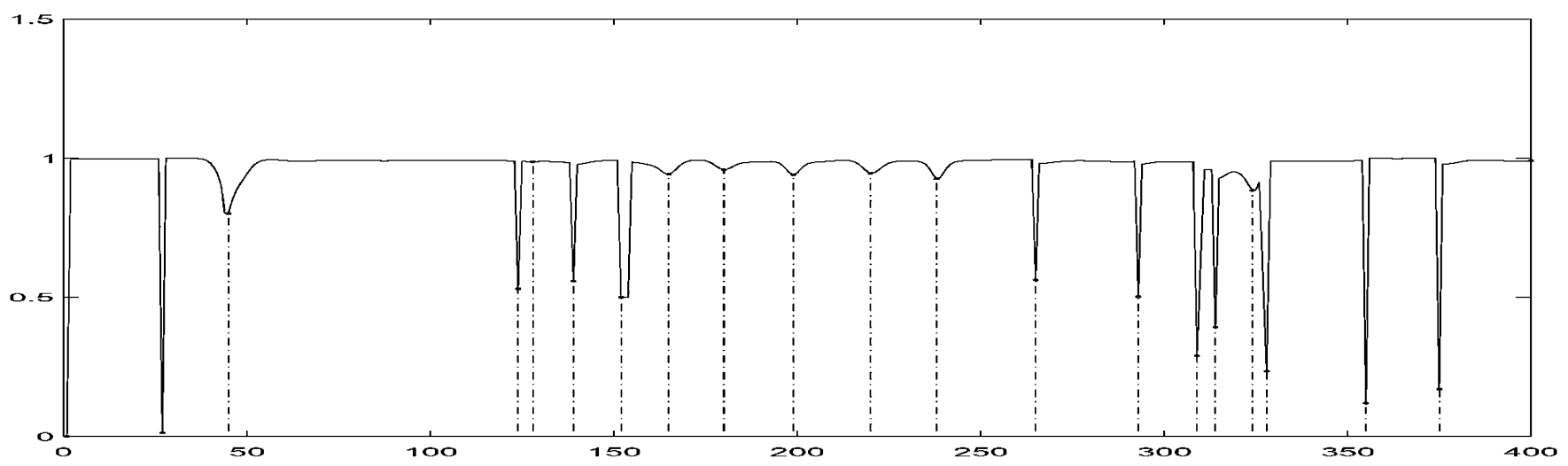

(b)

Fig. 3. (a) Shot detection using the fixed threshold method for a segment of the preview of Road Trip. Only first 400 frames are shown for convenient visualization. There are 19 shots identified by a human observer. (a) Fixed threshold method. Vertical lines indicate the detection of shots. Number of shots detected: 28; Correct: 19; False positive: 9; False negative: 0. (b) Shots detected by proposed method. Number of shots detected: 19; Correct: 19; False positive: 0; False negative: 0.

TABLE I

EXAMPLES OF SHOt DETECTION RESUlTS IN SOME PREVIEWS IN THE DATA SET

\begin{tabular}{l|c|c}
\hline \multicolumn{3}{c}{ Shot Detection Results } \\
\hline Movie & Recall & Precision \\
\hline \hline 24 Hours Party People & 0.96 & 0.84 \\
\hline Ali & 0.85 & 0.91 \\
\hline American Pie & 0.99 & 0.98 \\
\hline Americas Sweethearts & 0.95 & 0.92 \\
\hline Big Trouble & 0.89 & 0.92 \\
\hline Dracula 2000 & 0.95 & 0.96 \\
\hline The Fast and the Furious & 0.93 & 0.87 \\
\hline Hannibal & 0.94 & 0.86 \\
\hline The Hours & 0.88 & 0.99 \\
\hline Jackpot & 0.96 & 0.93 \\
\hline Kiss Of The Dragon & 0.97 & 0.90 \\
\hline Legally Blonde & 0.98 & 0.96 \\
\hline Mandolin & 0.91 & 0.95 \\
\hline Red Dragon & 0.96 & 0.91 \\
\hline Road Trip & 1.00 & 0.99 \\
\hline Rush Hour & 0.94 & 0.91 \\
\hline Sleepy Hollow & 0.96 & 0.89 \\
\hline Stealing Harvard & 0.98 & 0.95 \\
\hline The One & 0.95 & 0.86 \\
\hline The Others & 0.91 & 0.95 \\
\hline The Princess Diaries & 0.90 & 0.88 \\
\hline The World Is Not Enough & 0.96 & 0.83 \\
\hline The Tuxedo & 0.98 & 0.91 \\
\hline What Lies Beneath & 0.97 & 0.97 \\
\hline What Women Want & 0.96 & 0.94 \\
\hline & &
\end{tabular}

higher values for such a measure, and less visual disturbance would be expected for dramatic or romantic movies. To find visual disturbance, an approach based on the structural tensor computation is used which was introduced in [14]. The frames contained in a video clip can be thought of as a volume obtained by considering all the frames in time. This volume can be decomposed into a set of two 2-D temporal slices $I(x, t)$ and $I(y, t)$, where each is defined by planes $(x, t)$ and $(y, t)$ for horizontal and vertical slices, respectively. To find the disturbance in the scene, the structure tensor of the slices is evaluated, which is expressed as

$$
\boldsymbol{\Gamma}=\left[\begin{array}{ll}
J_{x x} & J_{x t} \\
J_{x t} & J_{t t}
\end{array}\right]=\left[\begin{array}{cc}
\sum_{w} H_{x}^{2} & \sum_{w} H_{x} H_{t} \\
\sum_{w} H_{x} H_{t} & \sum_{w} H_{t}^{2}
\end{array}\right]
$$

where $H_{x}$ and $H_{t}$ are the partial derivatives of $I(x, t)$ along the spatial and temporal dimensions, respectively, and $w$ is the window of support $(3 \times 3$ in our experiments $)$. The direction of gray level change in $w, \theta$, is expressed as

$$
R\left[\begin{array}{ll}
J_{x x} & J_{x t} \\
J_{x t} & J_{t t}
\end{array}\right] \quad R^{T}=\left[\begin{array}{cc}
\lambda_{x} & 0 \\
0 & \lambda_{t}
\end{array}\right]
$$

where $\lambda_{x}$ and $\lambda_{y}$ are the eigenvalues, and $R$ is the rotation matrix. With the help of the above equations, we can solve for the orientation angle $\theta$ as

$$
\theta=\frac{1}{2} \tan ^{-1} \frac{2 J_{x t}}{J_{x x}-J_{t t}} .
$$

When there is no motion in a shot, $\theta$ is constant for all pixels. With global motion (e.g., camera translation) the gray levels of 

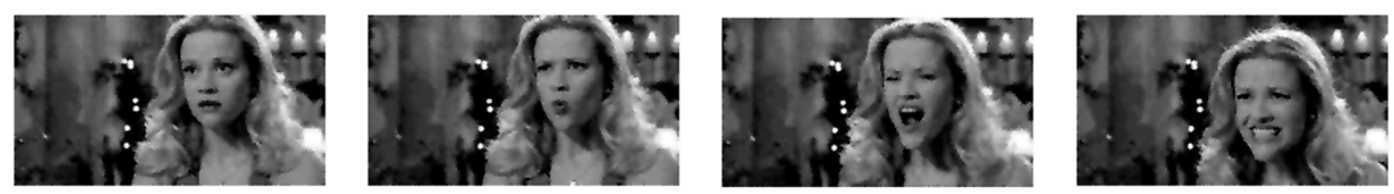

(a)
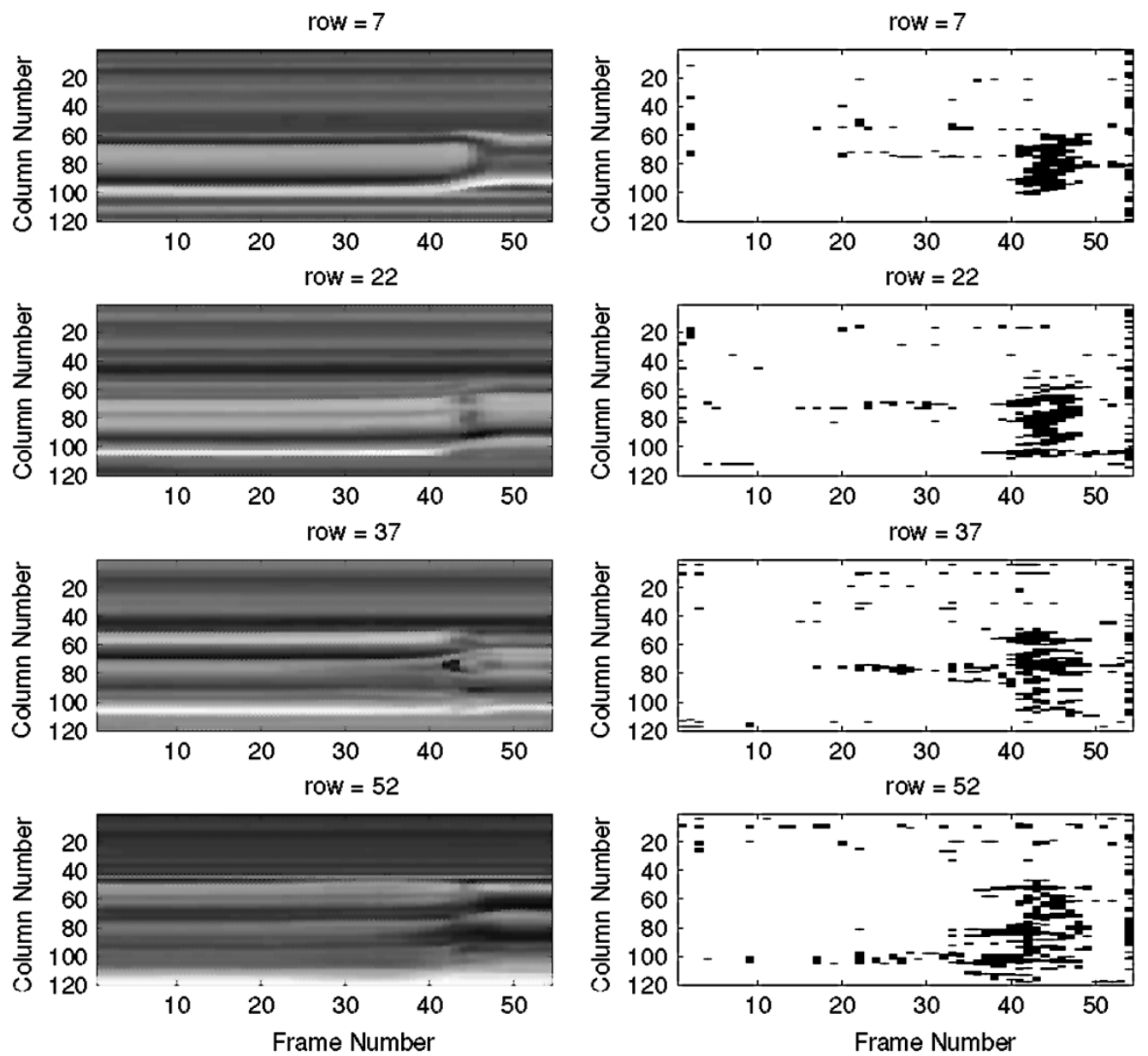

(b)

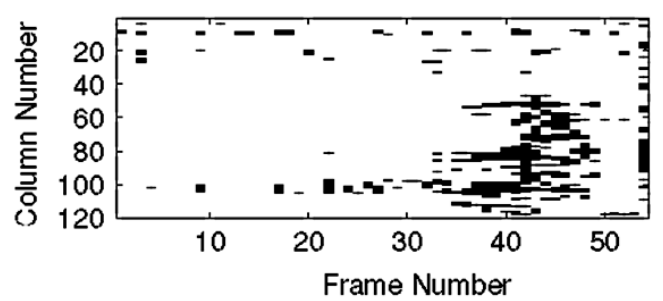

(c)

Fig. 4. Plot of visual disturbance. (a) Four frames of shots taken from the preview of Legally Blonde. (b) Horizontal slices for four fixed rows of a shot from the preview. Each column in the horizontal slice is a row of image. (c) Active pixels (black) in corresponding slices.

all pixels in a row change in the same direction. This results in equal or similar values of $\theta$. However, in the case of local motion, pixels that move independently will have different orientations. This can be used to label each pixel in a column of a slice as a moving or a nonmoving pixel.

The distribution of $\theta$ for each column of the horizontal slice is analyzed by generating a nonlinear histogram. Based on experiments, the histogram is divided into seven nonlinear bins with boundaries at $[-90,-55,-35,-15,15,35,55,90]$ o. The first and the last bins accumulate the higher values of $\theta$, whereas the middle one captures the smaller values. In a static scene or a scene with global motion all pixels have similar value of $\theta$ and therefore they fall into one bin. On the other hand, pixels with motion other than global motion have different values of $\theta$ and fall into different bins. The peak in the histogram is located and the pixels in the corresponding bin are marked as static, whereas the remaining ones are marked as active pixels. Next, a binary mask for the whole video clip is generated separating static pixels from active ones. The overall motion content is the ratio of moving pixels to the total number of pixels in a slice. Figs. 4 and 5 show motion content measure for two shots. Fig. 4 is a dialog shot taken from the movie Legally Blonde. On the other hand, Fig. 5 is a shot taken from a fight scene with high activity. Compare the density of moving pixels [black pixels in Fig. 5(c)] of both figures. It should be noted that the density of motion is much smaller for a nonaction shot as compared to an action shot.

\section{Lighting Key}

In the hands of an able director, lighting is an important dramatic agent. Generations of filmmakers have exploited luminance to evoke emotions, using techniques that are well studied and documented in cinematography circles [31]. A deliberate relationship exists, therefore, between the lighting and the genre of a film. 

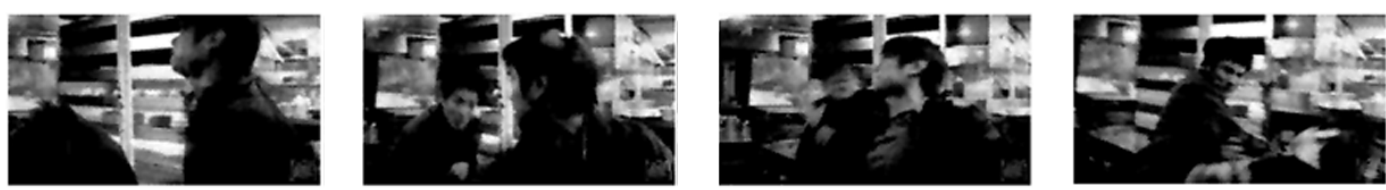

(a)
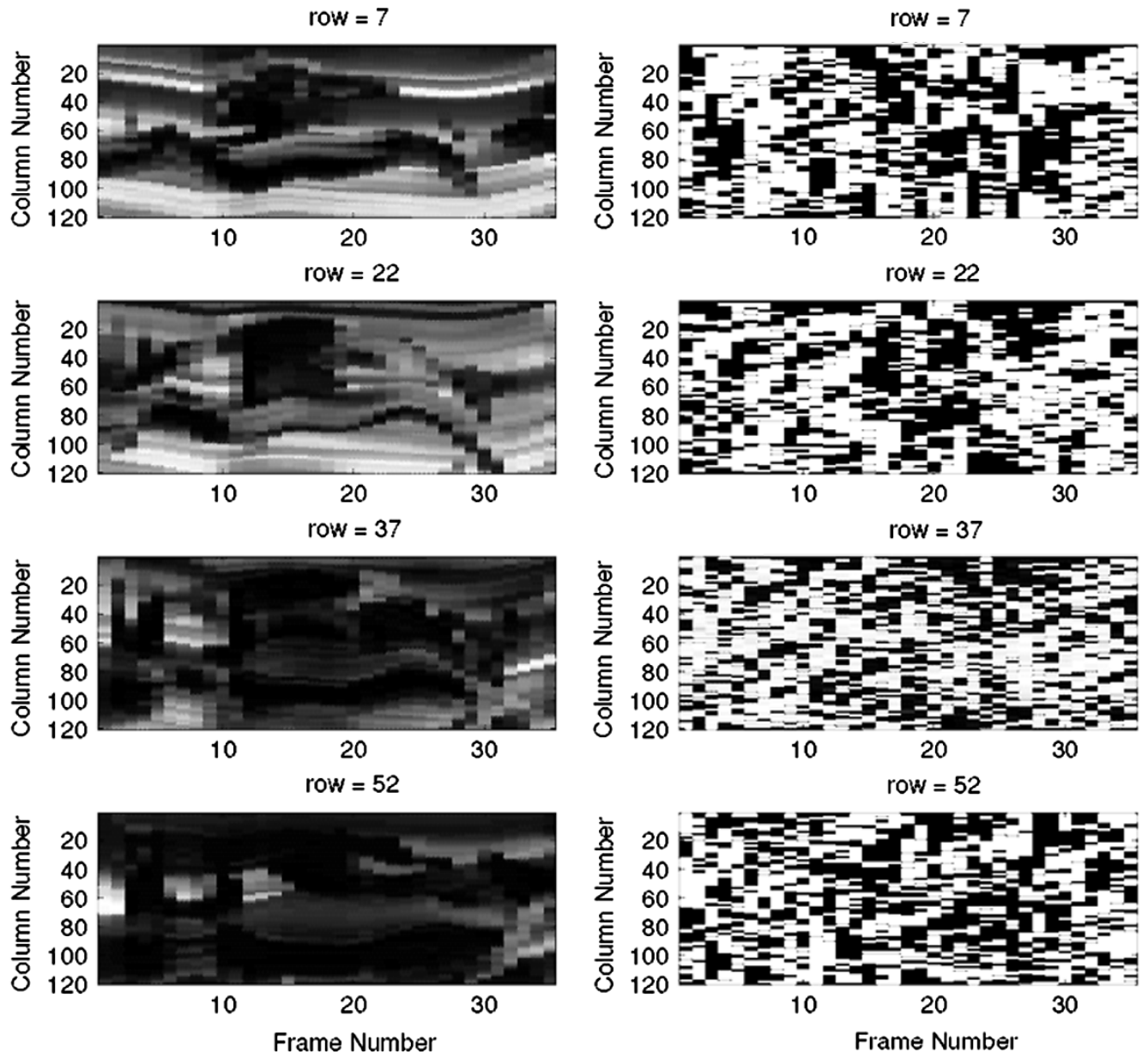

(b)

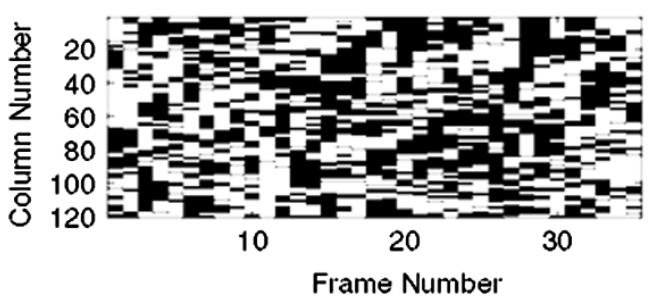

(c)

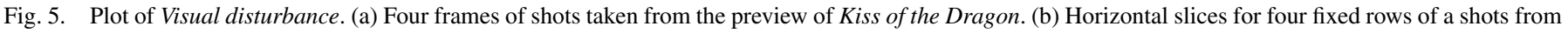
the preview. Each column in the horizontal slice is a row of image. (c) Active pixels (black) in corresponding slices.

In practice, movie directors use multiple light sources to balance the amount and direction of light while shooting a scene. The purpose of using several light sources is to enable a specific portrayal of a scene. For example, how and where shadows appear on the screen is influenced by maintaining a suitable proportion of intensity and direction of light sources. Lighting can also be used to direct the attention of the viewer to certain area of importance in the scene. It can also affect viewer's feeling directly regardless of the actual content of the scene. Reynertson comments on this issue, "The amount and distribution of light in relation to shadow and darkness and the relative tonal value of the scene is a primary visual means of setting mood" [24, pp. 107]. In other words, lighting is an issue not only of enough light in the scene to provide good exposure, but of light and shade to create a dramatic effect, consistent with the scene. In a similar vein, W. Rilla says "All lighting, to be effective, must match both mood and purpose. Clearly, heavy contrasts, powerful light and shade, are inappropriate to a light-hearted scene,
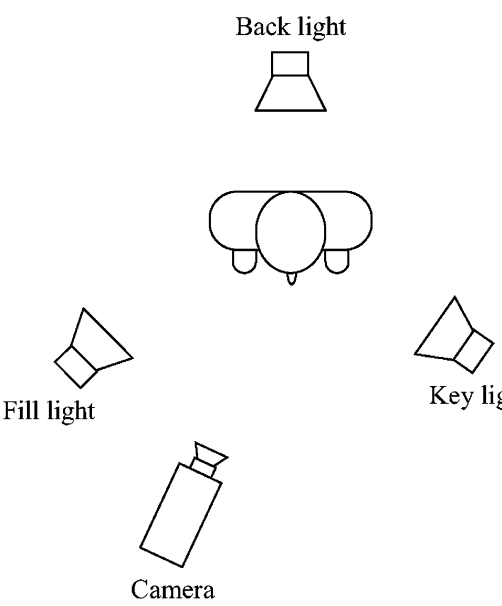

Key light

Fig. 6. Positioning of lights in a three-point lighting setup.

and conversely a flat, front-lit subject lacks the mystery which backlighting can give it" [25, p. 96]. 


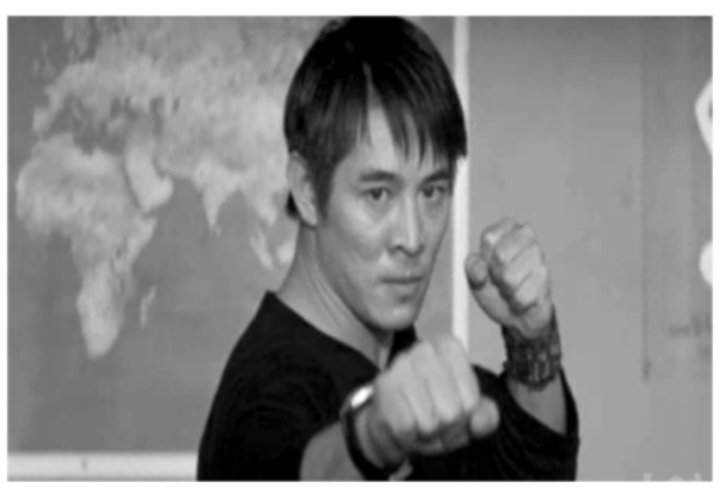

(a)

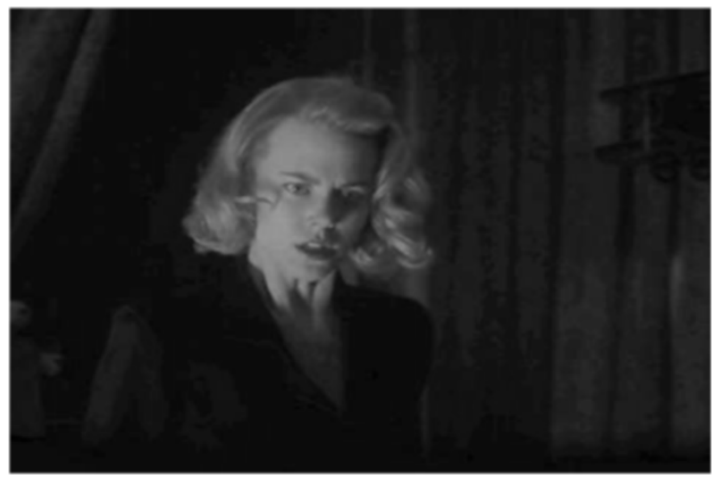

(c)

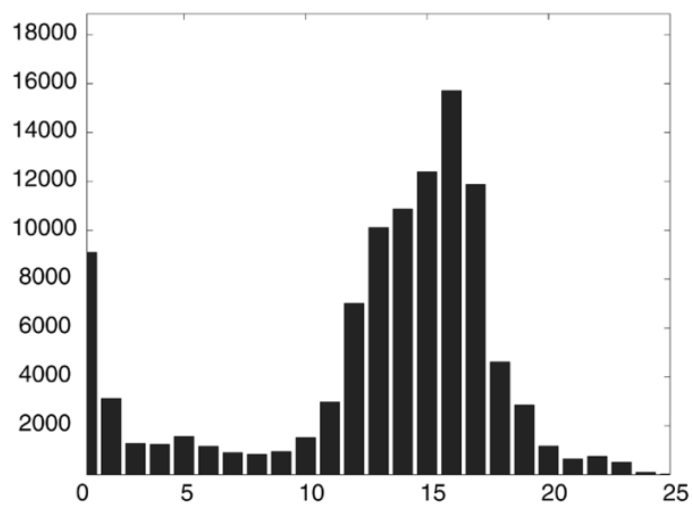

(b)

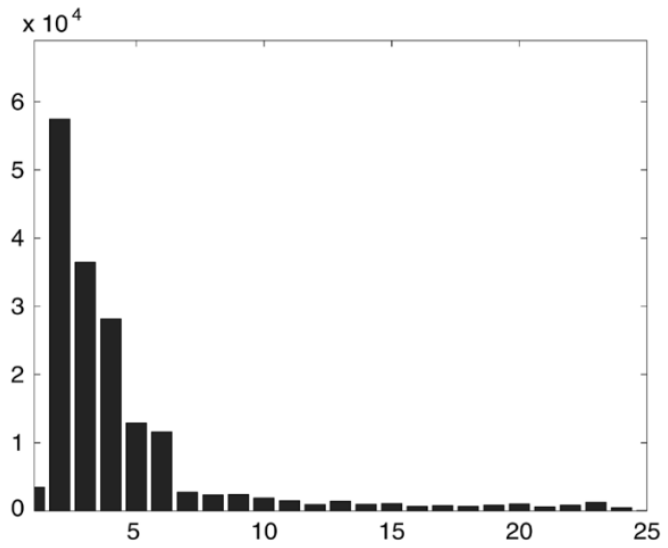

(d)

Fig. 7. Distribution of gray scale pixel values in (a) high-key shot (b) histogram and (c) low-key shot (d) histogram.

There are numerous ways to illuminate a scene. One of the commonly used methods in the film industry is called Three Point Lighting. As the name implies, this style uses three main light sources.

Keylight: This is the main source of light on the subject. It is the source of greatest illumination.

Backlight: This source of light helps emphasize the contour of the object. It also separates it from a dark background.

Fill-light: This is a secondary illumination source which helps to soften some of the shadows thrown by the keylight and backlight.

Fig. 6 shows how the light sources are placed with respect to the camera and the subject. With different proportions of intensity of each source, movie directors paint the scene with light and typify the situation of the scene. Thus, within the design phase of a scene there is deliberate correlation between scene context and the lighting of the scene. In film literature, two major lighting methods are used to establish such a relation between the context and the mood of the viewer, called low-key lighting and high-key lighting.

High-key lighting: High-key lighting means that the scene has an abundance of bright light. It usually has less contrast and the difference between the brightest light and the dimmest light is small. Practically, this configuration is achieved by maintaining a low key-to-fill ratio, i.e., a low contrast between dark and light. High-key scenes are usually contain action or are less dramatic. As a result, comedies and action films typically have high-key lighting [31, p. 32].

Low-key lighting: In this type, the background of the scene is generally predominantly dark. In low-key scenes, the contrast ratio is high. Low-key lighting is more dramatic and is often used in film noir or horror films.

Many algorithms exist that compute the position of a light source in a given image [21]. If the direction and intensity of the light sources are known, the key of the image can be easily deduced, and some higher level interpretation of the situation can be elicited. Unfortunately, for general scenes of the nature usually encountered in films, assumptions typically made in existing algorithms are violated, for example, single light source or uniform Lambertian surface. However, it is still possible to compute the key of lighting using simple computation. The brightness value of pixels in an image vary proportionally with the scene illumination and the surface properties of the observed object. Hence, a high-key shot, which is more illuminated than a low-key shot, contains a higher proportion of bright pixels. On the other hand, a low-key frame contains more pixels of lower brightness. This simple property has been exploited here to distinguish between these two categories. Fig. 7 shows the distribution of brightness values of high and low key shots. It can be roughly observed from the figure that for low-key frames, both the mean and the variance are low, whereas for high key frames the mean and variance are both higher. Thus, for a given key frame $i$ with $m \times n$ pixels in it, we find the mean $\mu$ and standard deviation $\sigma$ of the value component of the HSV space. The 


\section{Genre Membership of Data Set}

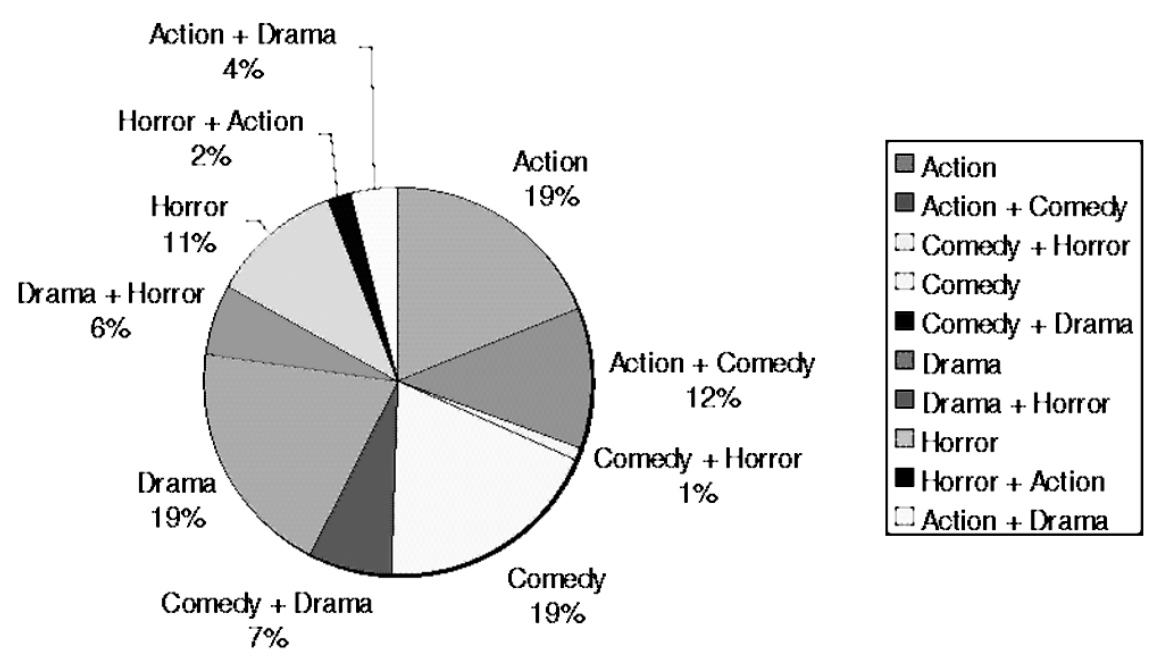

Fig. 8. Genre membership of data set. It should be noted that some films have a second genre, as well.

value component is known to correspond to brightness. A scene lighting quantity $\zeta_{i}(\mu, \sigma)$ is then defined as a measure of the lighting key of a frame

$$
\zeta_{i}=\mu_{i} \cdot \sigma_{i}
$$

In high-key frames, the light is well distributed which results in higher values of standard deviation and the mean, whereas in low-key shots, both $\mu$ and $\sigma$ are small. This enables us to formally interpret a higher level concept from low-level information, namely the key of the frame. In general, previews contain many important scenes from the movie. Directors pick the shots that emphasize the theme and put them together to make an interesting preview. For example, in horror movies, the shots are mostly low-key to induce fear or suspense. On the other hand, comedy movies tend to have a greater number of high-key shots, since they are less dramatic in nature. Since horror movies have more low-key frames, both mean and standard deviation values are low, resulting in a small value of $\zeta$. Comedy movies, on the other hand will return a high value of $\zeta$ because of high mean and high standard deviation due to wider distribution of gray levels.

\section{MeAn Shift Classification}

Thus far, we have discussed the relevance of various low-level features of video data, implying a formulation based on feature-space analysis. The analysis of the feature-space itself is a critical step that determines both the effectiveness and the practicality of the method. Even with a highly discriminating feature space, if the analysis is rule-based or imposes an unwarranted structure on the data (e.g., linear classifiers, elliptical shape, etc.), the possibility of extending or deploying the work becomes suspect. Extendibility, in particular, is a central aspect of this work, as we ultimately envision an interdependent, low-to-high level analysis toward semantic understanding. Although a multitude of techniques exist for the analysis of feature spaces (see [15] for a recent survey), most are unsuited

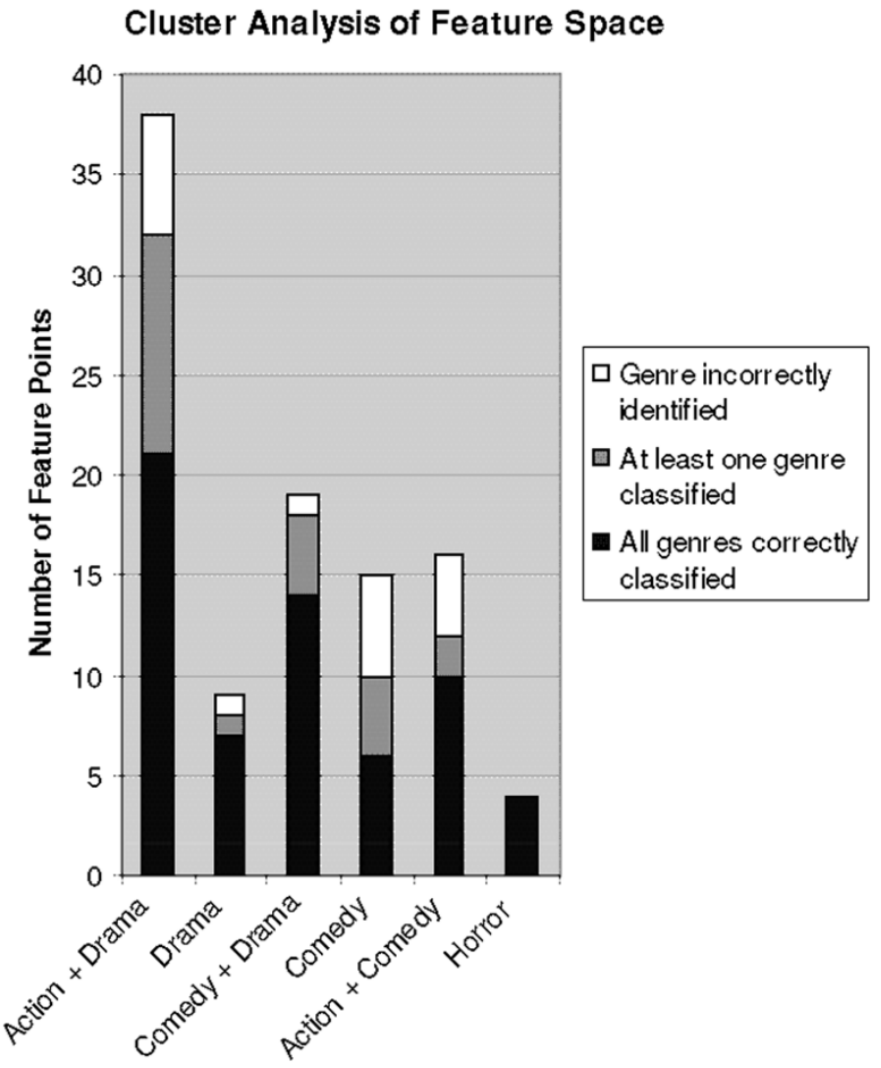

Fig. 9. Cluster analysis of feature space. Six clusters are observed in the data and each cluster is classified by its dominating genre.

for the analysis of real data. In contrast, the mean shift procedure has been shown to have excellent properties for clustering and mode-detection with real data. An in-depth treatment of the mean shift procedure can be found in [8]. Two salient aspects of mean shift based clustering that make it suited to this application is its ability to automatically detect the number of clusters and the fact that it is nonparametric in nature (and as a result does not impose regular structure during estimation). Since the four-dimensional (4-D) feature space is composed of the lighting-key, 


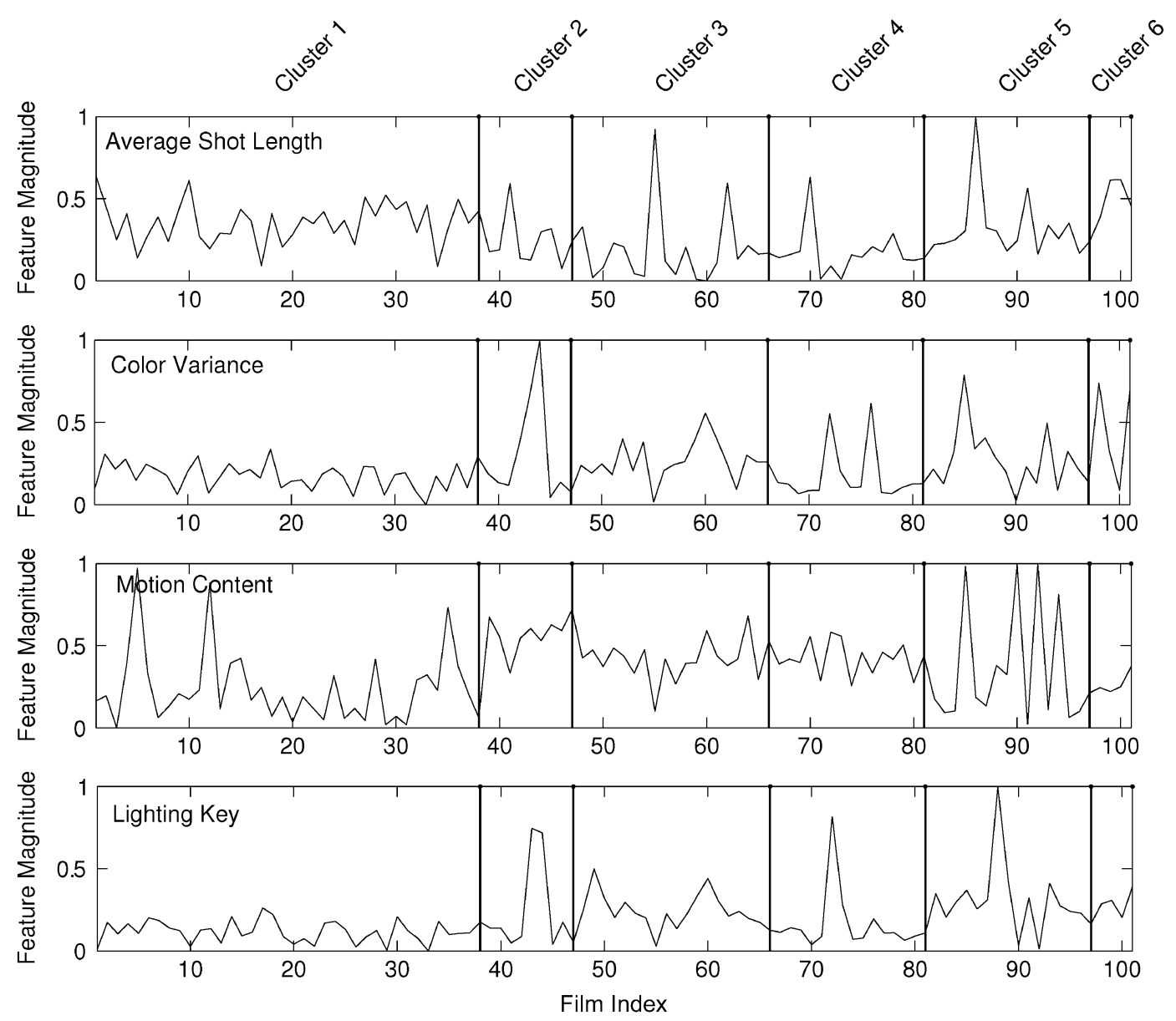

Fig. 10. Profiles of each feature. The films are indexed according to their association with each cluster.

average shot length, motion content, and color variance, we employ a joint domain representation. To allow separate bandwidth parameters for each domain, the product of four univariate kernels define the multivariate kernel, that is

$$
K(\mathbf{x})=\frac{C}{h_{1} h_{2} h_{3} h_{4}} \prod_{i=1}^{4} k\left(\frac{x_{i}^{2}}{h_{i}}\right)
$$

where $x_{i}, i=1$ to 4 , corresponds to the average shot length, color variance, motion content, and lighting key, respectively, $h_{i}$ are their corresponding bandwidth parameters, and $\mathrm{C}$ is a normalization constant. A normal kernel is used, giving a mean shift vector of

$$
\begin{aligned}
\mathbf{m}_{n, N}\left(\mathbf{y}_{j}\right) & =\mathbf{y}_{j+1}-\mathbf{y}_{j} \\
& =\frac{\sum_{i=1}^{4} \mathbf{x}_{i} \exp \left(\left\|\frac{x-x_{i}}{h_{i}}\right\|^{2}\right)}{\sum_{i=1}^{4} \exp \left(\left\|\frac{x-x_{i}}{h_{i}}\right\|^{2}\right)}-\mathbf{y}_{j} .
\end{aligned}
$$

Mean shift clustering provides a means to analyze the feature space without making arbitrary assumptions and lets the data define the probabilities of membership, so to speak. This formulation enables us to examine how well the computable features discriminate between the high-level labels known a priori. As a result, an exemplar based labeling system is also facilitated, since if there are consistent clusters and the label of one within the cluster is known, labels can be assigned to the rest.

\section{RESUlTS AND DiscusSION}

We have conducted extensive experiments on just over a hundred film previews. These previews were obtained from the Apple website, [2]. For each preview, video tracks were analyzed at a frame rate of $12 \mathrm{f} / \mathrm{s}$. The results of our experiments indicate interesting structure within the feature space, implying that a mapping does indeed exist between high-level classification and low-level computable features. We identified four major genres, namely, Action, Comedy, Horror, and Drama. We will first present our data set and the associated ground truth, followed by experimental results and discussion.

To investigate the structure of our proposed low-level feature space we collected a data set of 101 film previews, the ground truth of which is graphically displayed in Fig. 8. As mentioned earlier, classifying movies into binary genres (as opposed to mixed genres) is unintuitive, since modern cinema often produces films with more than one theme (presumably for both aesthetic and commercial reasons). Thus, we study multiple memberships both within the ground truth and the output of the proposed method. We performed mean shift classification over all the data points in the feature space, and studied the statistics of each cluster that formed. In the following discussion, we refer to the ground truth genres as labels and the cluster genres as classes.

The data formed six clusters in the 4-D feature space, the analysis of which are displayed in Fig. 9. Each cluster was 


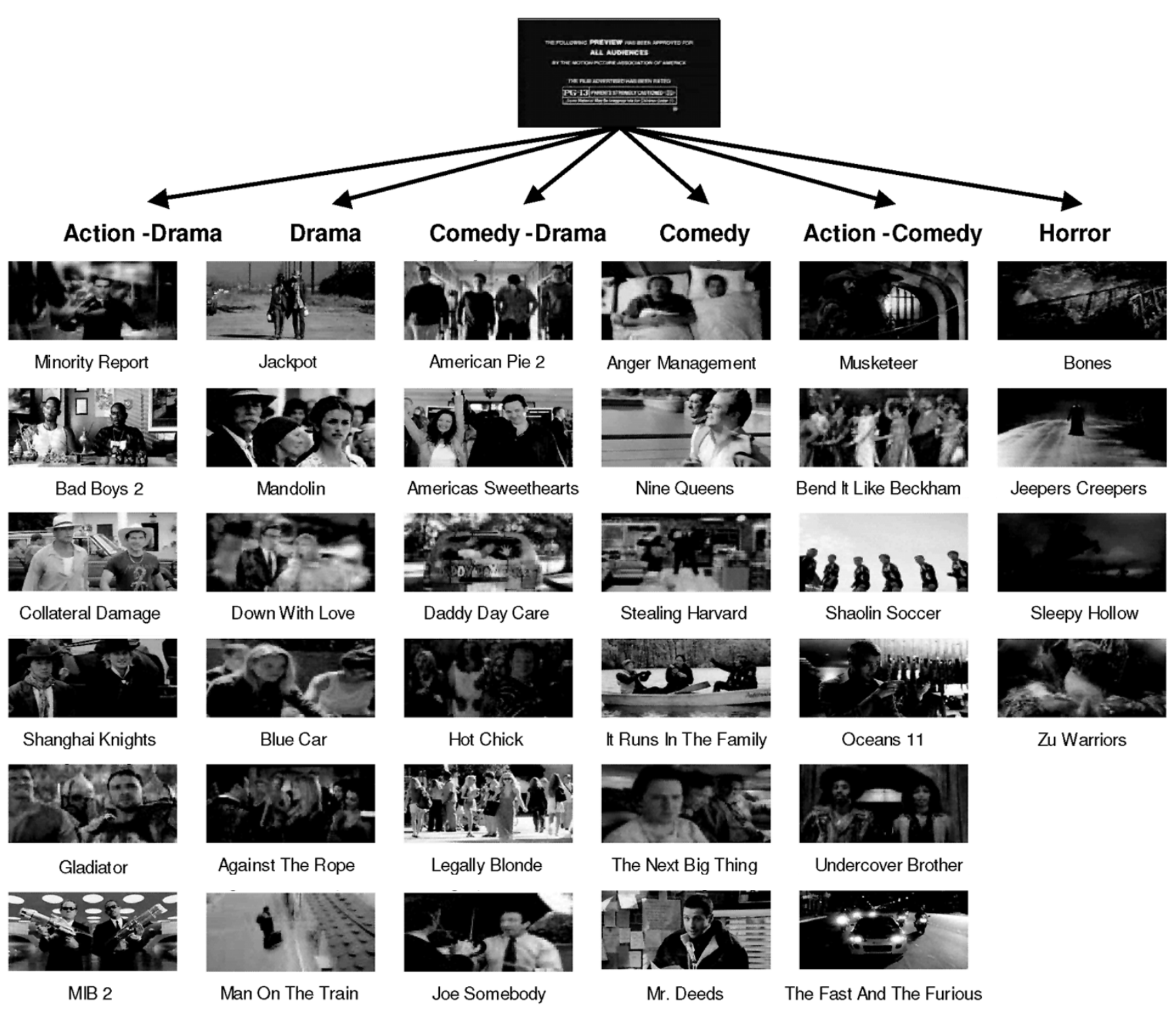

(a)

Fig. 11. (a) Examples of film previews in the data set with their associated classes.

assigned the label of the "dominating genres" in the cluster. We analyzed each cluster formed, counting the number of films 1) with all genres correctly identified; 2) at least one genre correctly identified; and 3) no genre correctly identified. The first (and the largest) cluster that was identified was the Action-Drama cluster, with 38 members. Although, only five movies were labeled Action-Dramas in the ground truth, all five appeared within this cluster. Moreover, the remaining points within this cluster were composed of ten films labeled as action films, and six films labeled as dramas. Eleven films with at least one genre labeled as drama or action were also observed in this cluster. The majority of the outliers (five out of six) came from the Horror genre.

The dominating genre in the second cluster was drama, with nine members. Nineteen films were labeled dramas in the ground truth, and eight of them were classified in this cluster. Only one outlier was observed within this cluster, Darkness Falls, which was labeled Horror in the ground truth. The third cluster was classified as Comedy and Drama, with 19 members.
Seven films were initially labeled as comedic dramas in the ground truth, and four of these seven were classified in this cluster. The cluster contained eight films labeled as comedies and two films labeled as dramas. The only outlier was the horror film, Session 9. The fourth cluster, classified as comedy, contained the highest percentage of outliers. Of the 15 films in the cluster, six were labeled comedies, four had at least one genre labeled as comedy, and five were incorrectly identified. The fifth cluster was classified as Action and Comedy and had a population of 16. Four films in this cluster were labeled as Action Comedies, five were action movies, and one was a comedy. In the last cluster, classified as Horror, we had four horror films grouped together. This small cluster can be seen as the only successful cluster of horror films, showing that while our features are not sufficiently discriminating for all horror films, it captures some of the structure that exists. Since our feature space is 4-D, we cannot visually display the clustering. In order to give the reader some feeling of the results, Fig. 10 displays the "profile" of each feature. The films are indexed 
according to their association with each cluster. See Fig. 11 for thumbnails of some the film previews in the data set associated with their classes.

The total number of outliers in the final classification was 17 (out of 101). While this number cannot be interpreted as an $83 \%$ genre classification accuracy, it strongly supports the claim that a mapping exists between low-level video features and high-level film classes, as predicted by film literature. Thus, this domain provides a rich area of study, from the extension and application of this framework to scene classification, to the exploration of higher level features, and as the entertainment industry continues to burgeon, the need for efficient classification techniques is likely to become more pending, making automated film understanding a necessity of the future.

\section{CONCLUSION}

In this paper, we have proposed a method to perform highlevel classification of previews into genres using low-level computable features. We have demonstrated that combining visual cues with cinematic principles can provide powerful tools for genre categorization. Classification is performed using mean shift clustering in the 4-D feature space of average shot length, color variance, motion content, and the lighting key. We discussed the clustering thus obtained and its implications in the Results section. We plan to extend this work to analyze complete movies and to explore the semantics from the shot level to the scene level. We also plan to utilize the grammar of movie making to discover the higher level description of the entire stories. Furthermore, we are interesting in developing computable features for mid-level and high-level information, as an interdependent multilevel analysis is envisaged. The ultimate goal is to construct an autonomous system capable of understanding the semantics and structure of films, paving the way for many "intelligent" indexing and postprocessing applications.

\section{ACKNOWLEDGMENT}

The authors would like to thank L. Spencer for her useful comments and corrections.

\section{REFERENCES}

[1] B. Adams, C. Dorai, and S. Venkatesh, "Toward automatic extraction of expressive elements from motion pictures: Tempo," in Proc. IEEE Int. Conf. Multimedia and Expo, 2000, pp. 641-644.

[2] Apple [Online]. Available: http://www.apple.com/trailers/

[3] D. Arijon, Grammar of the Film Language. New York: Hasting House, 1976.

[4] Internet Movie Data Base [Online]. Available: http://www.imdb.com/

[5] A. B. Benitez, H. Rising, C. Jrgensen, R. Leonardi, A. Bugatti, K. Hasida, R. Mehrotra, A. M. Tekalp, A. Ekin, and T. Walker, "Semantics of multimedia in MPEG-7," in Proc. IEEE Int. Conf. Image Process., vol. 1, 2002, pp. 137-140.

[6] J. S. Boreczky and L. D. Wilcox, "A hidden Markov model framework for video segmentation using audio and image features," in Proc. IEEE Int. Conf. Acoustics, Speech, Signal Process., 1997, pp. 3741-3744.

[7] S. F. Chang, W. Chen, H. J. Horace, H. Sundaram, and D. Zhong, "A fully automated content based video search engine supporting spatiotemporal queries," IEEE Trans. Circuits Syst. Video Technol., no. 5, pp. 602-615, Sep. 1998.

[8] D. Comaniciu and P. Meer, "Mean shift: A robust approach toward feature space analysis," IEEE Trans. Pattern Anal. Machine Intell., vol. 24, no. 5, pp. 603-619, May 2002.
[9] Y. Deng and B. S. Manjunath, "Content-based search of video using color, texture, and motion," in Proc. IEEE Int. Conf. Image Process. 1997, pp. 534-537.

[10] N. Dimitrova, L. Agnihotri, and G. Wei, "Video classification based on HMM using text and faces," presented at the Eur. Conf. Signal Process., Tampere, Finland, 2000.

[11] S. Fischer, R. Lienhart, and W. Effelsberg, "Automatic recognition of film genres," in Proc. 3rd ACM Int. Multimedia Conf. Exhibition, 1995, pp. 367-368.

[12] N. Haering, "A framework for the design of event detections," Ph.D dissertation, Sch. Comp. Sci., Univ. Central Florida, Orlando, 1999.

[13] Informedia Project, Digital Video Library [Online]. Available: http://www.informedia.cs.cmu.edu

[14] B. Jahne, Spatio-tmporal Image Processing: Theory and Scientific Applications. New York: Springer-Verlag, 1991.

[15] A. K. Jain, R. P. W. Duin, and J. Mao, "Statistical pattern recognition: A review," IEEE Trans. Pattern Anal. Machine Intell., vol. 22, no. 1, pp. 4-37, Jan. 2000.

[16] V. Kobla, D. S. Doermann, and C. Faloutsos, "Videotrails: Representing and visualizing structure in video sequences," in Proc. ACM Multimedia Conf., 1997, pp. 335-346.

[17] C. Lu, M. S. Drew, and J. Au, "Classification of summarized videos using hidden Markov models on compressed chromaticity signatures," in Proc. ACM Int. Conf. Multimedia, 2001, pp. 479-482.

[18] J. Nam, M. Alghoniemy, and A. H. Tewfik, "Audio-visual content based violent scene characterization," in Proc. IEEE Int. Conf. Image Process., 1998, pp. 353-357.

[19] M. R. Naphide and T. S. Huang, "A probabilistic framework for semantic video indexing, filtering, and retrieval," IEEE Trans. Multimedia, vol. 3, no. 1, pp. 141-151, Mar. 2001.

[20] The Handbook of Multimedia Information Management, N. V Patel and I. K. Sethi, Eds., Prentice-Hall, New York, 1997.

[21] A. Pentland, "Finding the illuminant direction," J. Opt. Soc. Amer., pp. 448-455, Jul.-Sep. 1982.

[22] P. Perona and J. Malik, "Scale-space and edge detection using anisotropic diffusion," IEEE Trans. Pattern Anal. Machine Intell., vol. 12, no. 7, pp. 629-639, Jul. 1990.

[23] R. Qian, N. Haering, and I. Sezan, "A computational approach to semantic event detection," IEEE Comp. Vision Pattern Recognit., vol. 1, no. 6, pp. 200-206, Jun. 1999.

[24] A. F. Reynertson, The Work of the Film Director. New York: Hasting House, 1970.

[25] W. Rilla, A-Z of Movie Making, A Studio Book. New York: Viking, 1970.

[26] B. T. Truong, S. Venkatesh, and C. Dorai, "Automatic genre identification for content-based video categorization," in Proc. IEEE Int. Conf. Pattern Recognit., 2000, pp. 230-233.

[27] N. Vasconcelos and A. Lippman, "Statistical models of video structure for content analysis and characterization," IEEE Trans. Image Process., vol. 9, no. 1, pp. 3-19, Jan. 2000.

[28] H. D. Wactlar, "The challanges of continuous capture; Contemporaneous analysis, and customzed summarization of video content, 2001," presented at the Defining a Motion Imagery Research and Development Program Workshop,, Washington, D.C., Nov. 2001.

[29] W. Wolf, "Hiden Markov model parsing of video programs," in Proc. Int. Conf. Acoustics, Speech, Signal Process., 1997, pp. 2609-2611.

[30] B. L. Yeo and B. Liu, "Rapid scene change detection on compressed video," IEEE Trans. Circuits Syst. Video Technol., vol. 5, no. 6, pp. 533-544, Dec. 1999.

[31] H. Zettl, Sight Sound Motion: Applied Media Aesthetics, 2nd ed. New York: Wadsworth, 1990.

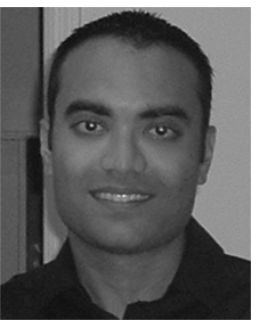

Zeeshan Rasheed received the B.S. degree in electrical engineering from NED University of Engineering and Technology, Karachi, Pakistan, in 1998 and the Ph.D. degree in computer science from the University of Central Florida, Orlando, in 2003.

He was awarded the Hillman Fellowship in 2001 for excellence in research in the Computer Science $\mathrm{Ph}$.D. program. His research interests include video understanding, video categorization, multimedia, human tracking, and real-time visual surveillance. 


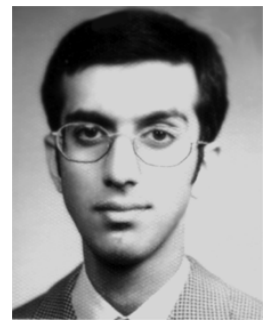

Yaser Sheikh (S'03) received the B.S. degree in electrical engineering from the Ghulam Ishaq Khan Institute of Engineering Sciences and Technology, Topi, Pakistan in 2001. He is currently working toward the $\mathrm{Ph}$.D. degree at the Vision Laboratory, University of Central Florida, Orlando.

His research interests include georegistration, video segmentation, compression, and action recognition. The current emphasis of his work is on correspondence across multiple camera systems.

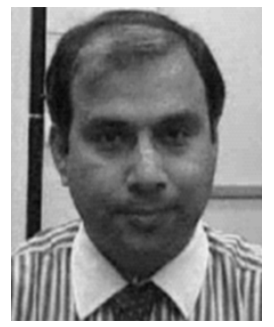

Mubarak Shah (M'86-SM'99-F'03) is a Professor of computer science, and the founding Director of the Computer Visions Laboratory, University of Central Florida, Orlando. He is a Researcher in computer vision, video computing, and video surveillance and monitoring. He has supervised several Ph.D., M.S., and B.S. degree students to completion and is currently directing $15 \mathrm{Ph} . \mathrm{D}$. and several B.S. students. He has published close to 100 articles in leading journals and conferences on topics including visual motion, tracking, video registration, edge and contour detection, shape from shading, stereo, activity, and gesture recognition, and multisensor fusion. He is coauthor of two books Motion-Based Recognition (New York: Kluwer, 1997) and Video Registration (New York: Kluwer, 2003), and an editor of international book series on Video Computing (New York: Kluwer, 2003).

Dr. Shah was an IEEE Distinguished Visitor speaker from 1997 to 2000, and is often invited to present seminars, tutorials, and invited talks all over the world. He received the Harris Corporation Engineering Achievement Award in 1999, the TOKTEN awards from UNDP in 1995, 1997, and 2000, the Teaching Incentive Program Awards in 1995 and 2003, the Research Incentive Award in 2003, and the IEEE Outstanding Engineering Educator Award in 1997. In addition, he was an Associate Editor of the Pattern Recognition and Machine Vision and Applications Journal, an Associate Editor the IEEE TRANSACTIONS ON PATTERN ANALYSIS AND MACHINE INTELLIGENCE (1998-2002), and a Guest Editor of the special issue of International Journal of Computer Vision on Video Computing. 